\title{
Ilha das Operações: Um Recurso Educacional Digital com Múltiplas Representações para a Compreensão do Campo Multiplicativo
}

\author{
Juscileide Braga de Castro ${ }^{1}$, Francisco Youri Miranda de Freitas ${ }^{1}$, Lidya de Lima \\ Monteiro Rufino ${ }^{1}$, Joyce da Silva Sousa ${ }^{1}$, Nathan Souza ${ }^{1}$, Márcia Duarte \\ Medeiros ${ }^{1}$, Maria de Fátima Souza ${ }^{1}$, José Aires de Castro Filho ${ }^{1}$ \\ ${ }^{1}$ Grupo de Pesquisa e Produção de Ambientes Interativos e Objetos de Aprendizagem - \\ PROATIVA. Instituto UFC Virtual, Universidade Federal do Ceará.

\footnotetext{
juscileidedvirtual.ufc.br; yourimiranda, lidyamont, joycedssousa,
} \\ ant.nathan15\}@gmail.com; \{marcia, fatimasouza, aires\}@virtual.ufc.br
}

\begin{abstract}
This article aims to present and describe the process of creating the Digital Educational Resource (DER) Ilha das Operations and its strategies to facilitate the learning process about situations in the multiplicative conceptual field. The DER was developed with HTML5, Java Script and CSS. From the pedagogical point of view, this RED is intended for the 4th year of Elementary School, according to the BNCC, and can be used inside or outside the classroom, for solving exercises and for diagnostic evaluation. With the support of gamification and an interactive interface with multiple representations, the user is invited to solve five random, quaternary situations that explore the idea of proportionality

Resumo. Este artigo tem como objetivo apresentar e descrever o processo de criação do Recurso Educacional Digital (RED) Ilha das Operações e suas estratégias para facilitar o processo de aprendizagem acerca de situações do campo conceitual multiplicativo. O RED foi desenvolvido com HTML5, Java Script e CSS. Do ponto de vista pedagógico, este RED destina-se ao $4^{\circ}$ ano do Ensino Fundamental, de acordo com a BNCC, podendo ser usando dentro ou fora de sala de aula, para resolução de exercícios e para avaliação diagnóstica. Com o suporte da gamificação e interface interativa com múltiplas representações, o usuário é convidado a resolver cinco situações randômicas, quaternárias, que exploram a ideia de proporcionalidade.
\end{abstract}

\section{Introdução}

Vários são os desafios encontrados no processo de ensino e de aprendizagem da Matemática. É comum verificarmos abordagens com ênfase em modelos de aplicação de fórmulas e com destaque nos exercícios práticos desvinculados da realidade do estudante [Magina, Santos e Merlini 2014]. Estas práticas não contribuem para a compreensão conceitual e a reflexão dos estudantes resultando em dificuldades e aversão à Matemática.

Indicadores como os do Sistema de Avaliação de Educação Básica (SAEB), apontam sucessos e dificuldades dos estudantes no âmbito da aprendizagem. Os resultados de avaliações realizadas em 2016 revelaram dificuldades na resolução de situações do campo multiplicativo, ou seja, que envolvem a multiplicação e a divisão. Segundo o Instituto Nacional de Estudos e Pesquisas Educacionais Anísio Teixeira (INEP), 69,39\% dos estudantes do $5^{\circ}$ ano do Ensino Fundamental, estão abaixo do nível 
6, em uma escala de proficiência que vai de 0 a 10 [Brasil 2017a]. De acordo com a matriz de proficiência do SAEB, estes estudantes não são capazes de: Resolver problemas de multiplicação com a noção de proporcionalidade (nível 6); Resolver problemas que de divisão exata ou a multiplicação de números naturais (nível 7); Resolver problemas que envolvem grandezas diretamente proporcionais (nível 8) [Brasil 2015].

Em concordância com a Base Nacional Comum Curricular (BNCC), situações do campo multiplicativo devem ser trabalhadas a partir do $2^{\circ}$ ano e por todo o Ensino Fundamental [Brasil 2017b]. Pesquisas [Castro 2016; Magina, Santos e Merlini; 2014; Vergnaud 1988] indicam que estas situações devem ser exploradas ponderando as diferentes ideias abordadas; as regras, os procedimentos e as relações envolvidas (funcional e escalar); assim como os diferentes tipos de representações (icônica, tabular, gráfica, dentre outras) mobilizadas pelos estudantes para ajudar na resolução.

Tyler, Prain e Peterson (2007, p. 317) explicam que "a aprendizagem de novos conceitos não pode ser separada da maneira de como aprender a representá-los e nem do que significam as representações usadas". Logo, os conceitos matemáticos adquirem sentido a partir de um conjunto de situações em que não se é possível isolar um único conceito. Os estudantes passam a compreender conceitualmente um conceito quando é capaz de mobilizá-lo em diferentes situações e representações.

Uma abordagem metodológica que pode ser utilizada pelos professores para trabalhar situações multiplicativas ou outros conteúdos curriculares é a que contempla Tecnologias Digitais da Informação e Comunicação (TDIC). Tendo em vista a crescente expansão das TDIC, evidencia-se a aplicação desses artefatos em sala de aula como potencial para a construção de conteúdos curriculares. Ademais, a BNCC indica a cultura digital como uma competência geral que precisa ser trabalhada nas escolas brasileiras [Brasil 2017b].

O uso de tecnologias digitais para fins educacionais pode trazer vantagens como: a facilidade de visualização e representação de gráficos; as simulações de situações reais; o trabalho em contextos investigativos; a produção de conteúdo e informação; dentre outras [Castro 2012]. Destacam-se ainda as vantagens de explorar as múltiplas representações, de forma dinâmica e interativa [Castro 2016].

A pesquisa realizada por Castro (2016) explorou, por meio de uma intervenção, a utilização e a produção de tecnologias digitais para a construção de conceitos relacionados ao campo multiplicativo, em especial, às situações quaternárias - proporção simples, múltipla e dupla [Magina, Santos e Merlini; 2014; Vergnaud 1988]. A pesquisadora verificou que o trabalho com múltiplas representações tem o potencial de contribuir com o desenvolvimento cognitivo, no entanto, relata que há poucos Recursos Educacionais Digitais (RED) que possam ser utilizados com esta finalidade.

Considerando o potencial das múltiplas representações e as dificuldades conceituais que os estudantes do Ensino Fundamental costumam ter diante da resolução de situações multiplicativas, desenvolvemos o RED Ilha das operações: em busca das pedras Múltiplis e Divisarions. Diante disso, o objetivo deste trabalho é apresentar e descrever o processo de criação e suas estratégias para facilitar o processo de aprendizagem acerca de situações do campo conceitual multiplicativo.

Esse artigo está dividido em quatro seções. A primeira, a introdução, na qual discutem-se problemas que justificam a produção do RED Ilha das operações, assim como 
sua relevância para as escolas. A seção seguinte relata o processo de desenvolvimento do RED, assim como a linguagem de programação utilizadas. Já a terceira seção traz uma descrição detalhada do RED. Por fim, tem-se as considerações finais.

\section{Processo de Desenvolvimento}

O RED Ilha das Operações ${ }^{1}$ : em busca das pedras Múltiplis e Divisarions foi desenvolvido por um grupo multidisciplinar composto por estudantes de graduação, professores da graduação e pós-graduação, bem como profissionais das áreas de design e programação, todos vinculados ao Grupo de Pesquisa e Produção de Ambientes Interativos e Objetos de Aprendizagem (PROATIVA). Tais profissionais constituíram duas equipes principais: pedagógica e técnica.

A equipe pedagógica, ficou responsável pela criação dos contextos, das atividades, dos textos, das narrativas e da incorporação dos elementos de gamificação, além dos conceitos matemáticos e dos feedbacks de interação com o usuário. Já a equipe técnica ficou encarregada das proposições de elementos gráficos, sonoros, fluidez das interações e ferramentas de programação. Destaca-se a integração entre as equipes como fator marcante durante as etapas de desenvolvimento. Para que houvesse organização e clareza na integração das tarefas desenvolvidas por ambas equipes, foi estabelecido um processo de desenvolvimento.

A primeira etapa consistiu da Ideação. Nela são definidos aspectos relacionados às habilidades e competências de acordo com as diretrizes da BNCC, o conteúdo a ser trabalhado e os possíveis elementos de gamificação a serem inseridos. Durante essa etapa foram definidos os aspectos centrais do RED, tais como seus objetivos pedagógicos, conteúdos e habilidades a serem contemplados e definição do público-alvo. Em relação aos aspectos da gamificação, o foco consistiu em analisar quais elementos dos jogos poderiam potencializar o interesse do usuário pela temática abordada, como por exemplo, a narrativa, os desafios propostos, as bonificações e os feedbacks instantâneos. Optamos por inserir elementos de gamificação neste RED, a fim de proporcionar a conexão e o engajamento dos estudantes na execução da proposta de atividade apresentada.

A segunda etapa consistiu da estruturação do RED, definindo aspectos relacionados à representatividade, à contextualização e com a seleção dos elementos de gamificação a serem incorporados no projeto de desenvolvimento.

$\mathrm{Na}$ terceira etapa buscou-se projetar/modelar sequências de navegabilidade, elementos de interação e gamificação. Finalizada a terceira etapa, iniciamos a etapa de implementação com o intuito de produzir um executável. Na etapa de implementação, três linguagens principais foram utilizadas, sendo estas: HTML5 (marcação), JavaScript (programação) e CSS (estilo). Foi utilizado como framework para desenvolvimento o AngularJS, mais especificamente o modelo angular-webpack-config, que facilitou a programação ao dividi-la em estados bem definidos. Para desenvolvimento foi utilizado também o $S A S S$, uma linguagem que pré-processa o $C S S$, ajudando na organização do estilo e diminuindo o tamanho dos arquivos que eram modificados durante $o$ desenvolvimento, ao passo que os reunia quando partiam para a produção. O tamanho do arquivo era algo importante para a equipe, visto que se pretende que este RED possa funcionar online e offline.

\footnotetext{
${ }^{1}$ Disponível em: https://mide-ilha-das-operacoes.netlify.com/
} 
Na etapa seguinte, de averiguação, verificamos a sequência de navegabilidade, os elementos de interação e se os objetivos definidos no início desse processo são atendidos. Por fim, a etapa de autenticação que corresponde a etapa de validação, consiste em avaliar se os objetivos pedagógicos foram atingidos da forma como foi projetado.

É importante ressaltar, que as duas primeiras etapas (ideação e organização) foram de responsabilidade da equipe pedagógica. A etapa de projetar/modelar foi desenvolvida pelas duas equipes. A etapa de implementação foi realizada apenas pela equipe técnica. Por fim, as etapas de averiguação e autenticação foram desenvolvidas em conjunto por ambas as equipes. Isto porque, à medida que se identificava alguma inconsistência no recurso, de imediato era reportada a equipe técnica e a mesma já providenciava a solução. A seção 3 apresenta os aspectos pedagógicos do Ilha das Operações.

\section{O RED Ilha das Operações}

O RED Ilha das Operações²: em busca das pedras Múltiplis e Divisarions pode ser classificado como um jogo, que tem como público-alvo os estudantes do $4^{\circ}$ ano do Ensino Fundamental ou estudantes de outros níveis que tenham dificuldades na resolução de situações do campo multiplicativo. Ele pode ser utilizado dentro ou fora da sala de aula, para resolução de exercícios e para avaliação diagnóstica.

O RED prevê o desenvolvimento de habilidades da BNCC da unidade temática Números, o que envolve a compreensão das operações e o desenvolvimento de diferentes estratégias, assim como a resolução de problemas com diferentes significados da multiplicação e da divisão [Brasil 2017b], (quadro 1).

Quadro 1 - Habilidades do RED Ilha das Operações

\section{Habilidades de Matemática}

(EF04MA04) Utilizar as relações entre adição e subtração, bem como entre multiplicação e divisão, para ampliar as estratégias de cálculo.

(EF04MA05) Utilizar as propriedades das operações para desenvolver estratégias de cálculo.

(EF04MA06) Resolver e elaborar problemas envolvendo diferentes significados da multiplicação (adição de parcelas iguais, organização retangular e proporcionalidade), utilizando estratégias diversas, como cálculo por estimativa, cálculo mental e algoritmos.

(EF04MA07) Resolver e elaborar problemas de divisão cujo divisor tenha no máximo dois algarismos, envolvendo os significados de repartição equitativa e de medida, utilizando estratégias diversas, como cálculo por estimativa, cálculo mental e algoritmos.

O RED apresenta como narrativa o cenário de uma ilha dividida em quatro partes e que tem uma montanha em seu centro. No topo desta montanha estão localizadas quatro pedras fundamentais que representam os quatro povos que habitam a ilha e simbolizam o equilíbrio daquelas terras. Os povos do norte e do sul continuam cooperando entre si, mas o medo de não sobreviver toma conta dos habitantes do leste e do oeste, com isso, suas respectivas pedras perdem energia, chegando a desaparecer, iniciando o processo de destruição da ilha. Porém, o guardião das pedras, o grande Matemágico, estava confiante, pois havia uma profecia muito antiga, na qual haveria um salvador.

\footnotetext{
${ }^{2}$ Vídeo demonstrativo disponível em: https://www.youtube.com/watch?v=Tv8iHAQaB90
} 
Nesse contexto, o jogador torna-se fundamental para restaurar o equilíbrio da ilha, de modo que ele encontre as pedras desaparecidas e as coloque de volta no topo da montanha, antes que a destruição da ilha aconteça. Para que essa missão seja realizada, é necessário resolver cinco situações, apresentadas de forma linear. Cada uma delas está relacionada a um povo em conflito (multiplicação e/ou divisão) e a alguma necessidade na ilha. Antes de iniciar o desafio e à medida que vai resolvendo novos, o usuário ganha itens que serão usados na resolução das situações seguintes e que farão parte de seu inventário. $\mathrm{O}$ guardião Matemágico será o guia durante todo o processo de recuperação das pedras Múltiplis e Divisarions.

Todas as situações deste RED exploram a ideia de proporcionalidade, com grandezas diretamente proporcionais, sendo, portanto, situações com relações quaternárias, ou seja, envolvendo quatro termos [Castro 2016; Vergnaud 1988]. Devido a estas características, foi possível explorar representações textuais, icônicas e tabulares na interface de resolução das situações. Segundo Castro (2016), ao fazer relações entre estas três representações, o usuário pode perceber as relações funcionais ${ }^{3}$ e as escalares ${ }^{4}$.

Conforme pode-se observar na figura 1, além da situação escrita, têm-se a representação das quantidades envolvidas (representação icônica), assim como uma tabela com as informações presentes na situação. Destaca-se ainda que os textos em azul referem-se às quantidades das grandezas envolvidas, variando a cada novo jogo, pois foram programados para obedecer a intervalos randômicos, variáveis para cada desafio.

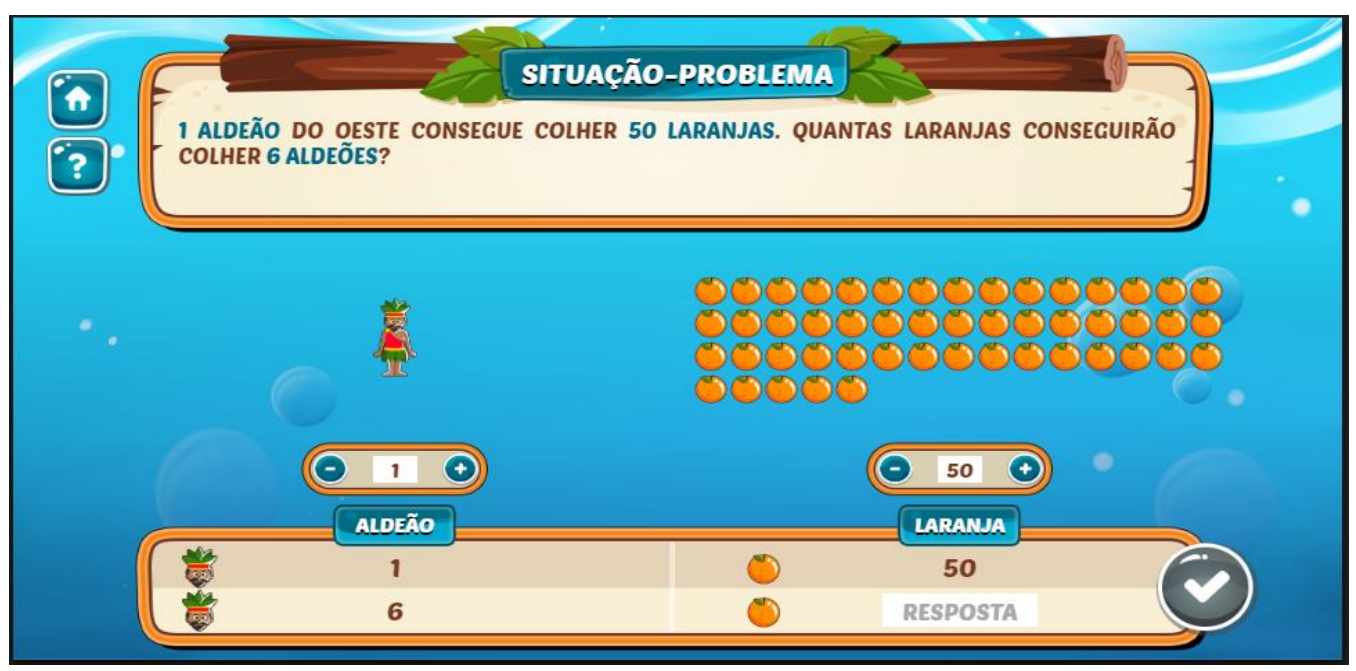

Figura 1. Primeira situação dos desafios para conquistar as pedras

Para obter o resultado o usuário pode interagir com as imagens ou com a tabela, de modo a perceber que se um aldeão colhe 50 laranjas, dois aldeões, com o mesmo ritmo de trabalho, colherão 100 laranjas, tendo-se uma relação de 50 laranjas por aldeão.

Apesar de todas as cinco situações explorarem a ideia de proporcionalidade, elas se diferenciam pelo nível de dificuldade, pelos eixos (proporção simples ou múltipla), pelas classes (um para muitos ou muitos para muitos), por possíveis operações mobilizadas (tipo) em sua resolução ou ainda pelas relações estabelecidas (quadro 2).

\footnotetext{
${ }^{3}$ Relação entre duas grandezas, o que remete a compreensão da relação fixa existente entre as quantidades envolvidas. Exemplo: a relação entre a quantidade de laranjas colhidas por aldeão.

${ }^{4}$ Relação dentro de uma mesma grandeza.
} 
Quadro 2 - Resumo das situações exploradas no RED

\begin{tabular}{|l|l|l|l|l|}
\hline Situação & Eixo & Classes & Tipo & Relações \\
\hline Situação 1 & Proporção simples & Um para muitos & Multiplicação & laranjas e aldeões \\
\hline Situação 2 & Proporção múltipla & Um para muitos & Multiplicação & caixote, caixa e laranjas \\
\hline Situação 3 & Proporção simples & Um para muitos & Divisão por partes & caixote e laranja \\
\hline Situação 4 & Proporção simples & Um para muitos & Divisão por cotas & cercado e cavalo \\
\hline Situação 5 & Proporção simples & Muitos para muitos & Multiplicação e divisão & cavalo e caixa \\
\hline
\end{tabular}

As classificações do quadro 2 estão em conformidade com as discutidas por Castro (2016) e por Magina, Santos e Merlini (2014). Para maiores detalhes das situações e das possibilidades pedagógicas do RED, sugerimos acessar o guia do professor ${ }^{5}$, documento criado para orientar o professor para a utilização do RED em sala de aula.

Ao resolver as situações propostas, o usuário vai recebendo itens para ser acrescentado ao seu inventário e sendo convidados a resolverem novos desafios. Dentre os itens têm-se materiais como caixas, caixotes, cercados, que serão utilizados para auxiliar o usuário na resolução de situações seguintes; assim como o mapa com a localização das pedras e as pedras perdidas - Múltiplis ou Divisarions.

O desafio final envolve as operações de multiplicação e de divisão com relação muito par muitos e pode ser considerada a situação mais difícil do RED (Figura 2).

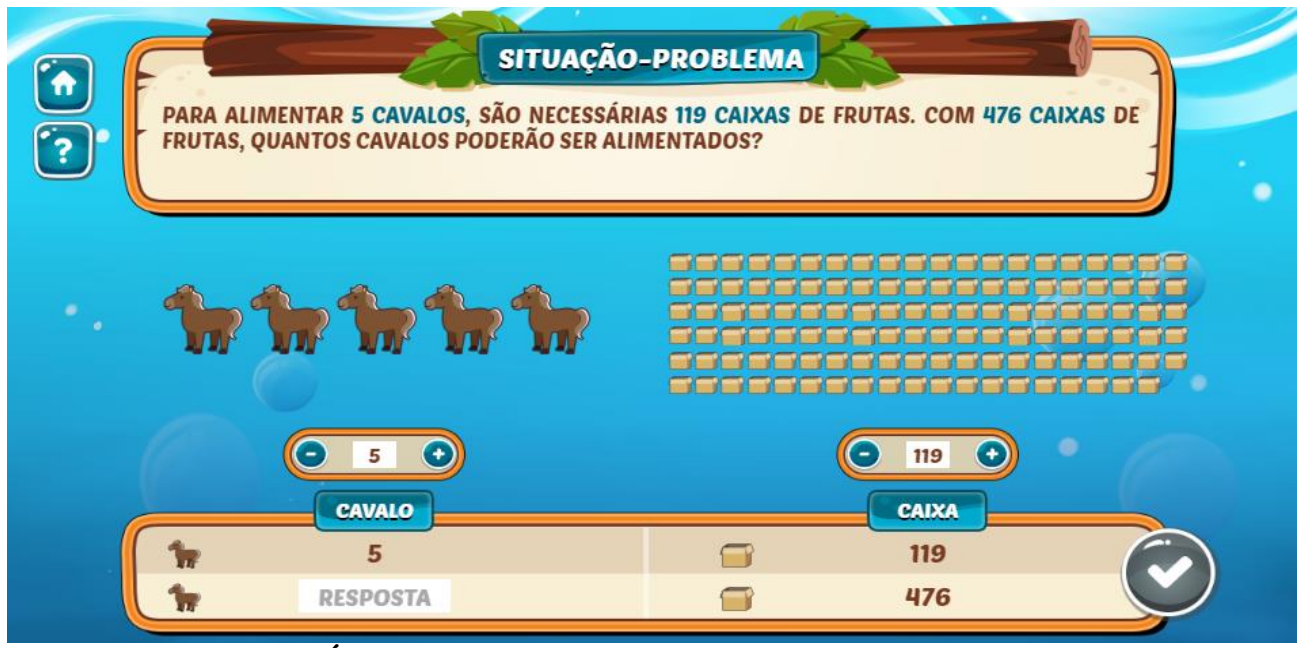

Figura 2. Última situação dos desafios para conquistar as pedras

Na Figura 2 tem-se 119 caixas para 5 cavalos. O usuário deve perceber que, neste caso, como a quantidade de caixas é 4 vezes maior $(476 / 119=4)$, logo a quantidade de cavalos também deve ser 4 vezes maior $(5 \times 4=20$ cavalos $)$. Ainda que o usuário possa encontrar o resultado manipulando a quantidade de representações icônicas, o professor poderá mediar e questionar as relações envolvidas. Ao finalizar o desafio, o usuário consegue devolver as pedras em seus lugares de origem, estabelecer a paz entre os povos e restaurar o equilíbrio na Ilha das Operações.

\footnotetext{
${ }^{5}$ Disponível em: https://plataformaintegrada.mec.gov.br/recurso?id=338674\&name=Ilha $\% 20$ das $\% 20$ Opera $\% \mathrm{C} 3 \% \mathrm{~A} 7 \% \mathrm{C} 3$ \%B5es
} 


\section{Considerações Finais}

O uso de RED na Educação como forma de amenizar os obstáculos encontrados nos processos de ensino e de aprendizagem faz com que haja uma preocupação constante em se repensar o modo como esses recursos devem ser apresentados e organizados. Com base nessa reflexão, foi desenvolvido o RED Ilha das Operações que busca conciliar um modo mais dinâmico e mais contextualizado de se organizar o conteúdo a ser trabalhado. No âmbito do dinamismo, buscou-se suporte na gamificação como forma de agregar valor ao material desenvolvido e engajamento do usuário, trazendo elementos desafiadores, temporalidade e bonificações. Já na esfera da contextualização, buscou-se pensar em cenários e situações que estimulassem a reflexão dos estudantes, além de compreensão conceitual por meio de uma interface explorando as múltiplas representações.

O RED Ilha das Operações buscou conciliar um contexto instigante associado a gamificação, tendo este último, o papel de empregar não somente o dinamismo peculiar dos jogos, mas também de estimular, de modo indireto, a compreensão e a aprendizagem dos conceitos matemáticos, relacionados às estruturas multiplicativas.

O RED Ilha das Operações é um protótipo, estando em processo de validação junto ao público-alvo. Esta validação conta com a aplicação do recurso com alunos do $4^{\circ}$ ano do Ensino Fundamental e com professores que ensinam matemática. Espera-se que a utilização desse recurso em sala de aula auxilie professores e alunos a minimizar as dificuldades encontradas no ensino e na aprendizagem dos conceitos relacionados ao campo conceitual das estruturas multiplicativas.

\section{Referências}

Brasil (2017a) "Base Nacional Comum Curricular". Ministério da Educação, Secretaria da Educação Básica, Brasília, DF: SEB.

Brasil (2017b) “SAEB 2017” Ministério da Educação. Brasília, DF: INEP.

Brasil (2015) "Escala de proficiência de Matemática - $5^{\circ}$ ano do Ensino Fundamental", Brasília, DF: INEP.

Castro, J. B. (2016) “Construção do conceito de covariação por estudantes do Ensino Fundamental em ambientes de múltiplas representações com suporte das tecnologias digitais" 2016. 275 f. Tese (Doutorado) - Curso de Doutorado em Educação Brasileira, Universidade Federal do Ceará, Fortaleza. Disponível em: http://www.repositorio.ufc.br/handle/riufc/15908 Acesso em 03 de maio de 2020

Castro, J. B. (2012) "A utilização de objetos de aprendizagem para a construção e compreensão de gráficos estatísticos". Dissertação (Mestrado) - Programa de Pós Graduação em Educação Brasileira, Universidade Federal do Ceará, Fortaleza. Disponível em: http://www.repositorio.ufc.br/handle/riufc/7341 Acesso em 03 de maio de 2020

Magina, S. P.; Santos, A.; Merlini, V. L. (2014) O raciocínio de estudantes do Ensino Fundamental na resolução de situações das estruturas multiplicativas. "Ciência \& Educação”, Bauru, v. 20, n. 2, p. 517-533.

Tytler, R.; Prain, V.; Peterson, S. (2007) Representational issues in students learning about evaporation. "Research in Science Education", v. 37, p. 313-331.

Vergnaud, G. (1988) Multiplicative structures. In: Hiebert, H.; Behr, M. "Research Agenda in Mathematics Education": number concepts and operations in the Middle Grades. Hillsdale, NJ: Lawrence Erlbaum, p. 141-161. 